\title{
Small-Town America's Despair: Infected Substance Users Needing Outpatient Parenteral Therapy and Risk Stratification
}

Ulas M. Camsari ${ }^{1}$, Claudia R. Libertin ${ }^{2}$

1. Department of Psychiatry \& Psychology, Mayo Clinic, Rochester, MN, USA, Rochester, MN, USA 2. Internal Medicine, Mayo Clinic, Jacksonville, FL

$\square$ Corresponding author: Ulas M. Camsari, camsarim@yahoo.com

Disclosures can be found in Additional Information at the end of the article

\section{Abstract}

\section{Background}

An active intravenous substance use disorder is often the primary cause of infectious diseases in this population of users and creates a barrier to successful parenteral antimicrobial management. The dilemma is compounded by dramatically limited resources in small US towns.

\section{Methods}

This retrospective review from January 2014 through July 2016 aimed to develop a risk stratification approach to aid rural healthcare providers in determining who among patients with addictive disorders could safely be discharged for outpatient antimicrobial therapy with a peripherally inserted central catheter (PICC).

\section{Results}

The high-risk group had a greater likelihood of noncompliance with antimicrobial therapy completion, as well as subsequent illicit drug use during that time frame, compared with the moderate- and low-risk groups. The low-risk group and most of the moderate-risk group could be safely discharged into the community with PICC lines.

\section{Conclusions}

Key in the risk stratification proposal was identifying risk behaviors and determining their degree. Such information provides pivotal delineators in developing risk stratification criteria.

Received 06/28/2017

Review began 07/11/2017 Review ended 07/28/2017 Published 08/18/2017

(c) Copyright 2017

Camsari et al. This is an open access article distributed under the terms of the Creative Commons Attribution License CC-BY 3.0., which permits unrestricted use, distribution, and reproduction in any medium, provided the original author and source are credited.
Categories: Psychiatry, Infectious Disease, Public Health

Keywords: : ivdu and opat, risk stratification of ivdu and opat, rural medicine, rural opat care, small town plights

\section{Introduction}

Guidelines on the management of people with intravenous substance use disorders, in clinical settings of severe systemic infectious diseases (IDs), who require parenteral antimicrobial therapy, are limited. Management is a challenge everywhere but especially in under-served rural communities, where resources are scarce, including ambulatory care units, transportation, finance, and even specialists. The available guidelines focus on antimicrobial therapy [1-3], yet 
offer little or no guidance on the overall treatment of patients who have intravenous substance use disorders. An active use disorder is often the primary cause of the ID and creates an important barrier to the success of the treatment and remission of the ID. Tools are needed to aid providers in identifying who could safely receive outpatient antimicrobial therapy (OPAT) in a clinical setting of intravenous drug use (IVDU) in rural settings.

Approximately $20 \%$ of the US population lives in rural areas. Yet, clinical practice in the rural United States can be challenging [4]. Clinical specialists, healthcare facilities, transitional care providers, and substance abuse treatment programs are scarce [5]. Compared with urban dwellers, persons living in rural areas have more health-related disparities. These effects include poorer health, more behaviors that place a person's health at risk, and limited access to health resources [6]. Substance use disorders, particularly opioid use disorder, have greater prevalence in rural United States [7-10]. In this article, we develop a risk stratification approach similar to approaches used in other disciplines [11-12] to aid rural health care providers in determining which persons with addictive disorders can safely be reviewed for OPAT.

\section{Materials And Methods}

This study was approved by the Mayo Clinic Institutional Review Board. A retrospective chart review was conducted from January 2014 through July 2016 at Mayo Clinic Health System in Waycross, Georgia, a 200-bed rural hospital with a daily bed occupancy rate of less than 100. It is an affiliate of Mayo Clinic in Jacksonville, Florida. We reviewed all cases that required parenteral, antimicrobial therapy in the outpatient setting as recommended by the ID specialist. The ID specialist screened all patients who may possibly be discharged from the hospital with a peripherally inserted central catheter (PICC) for drug and severe alcohol use. All infected patients who had a positive drug use history and needed a PICC line for discharge were referred for drug addiction consultation. Psychiatric consultations are a routine part of that practice when drug addiction could affect a patient's outcome. Patients undergo a comprehensive evaluation by a psychiatrist trained in addiction subspecialty and are given the appropriate diagnosis for their addictive disorder, according to the Diagnostic and Statistical Manual of Mental Disorders (Fifth Edition) (DSM-V) [13]. A psychosocial risk profile was created for each case. That profile was used in decision-making around the patient's disposition, venue of treatment, and recommendations on the ID diagnosis and addictive disorder concurrently. The only inclusion criteria implemented beyond time restrictions were that the person had to have an IVDU disorder, be evaluated by both specialists, and be in need of OPAT.

\section{Psychosocial risk evaluation}

Psychosocial risk evaluations by liaison psychiatrists focusing on patient safety and treatment outcomes are an established practice in assessing the risk levels of candidates for invasive procedures or operations, such as solid organ transplant, left ventricular assist device, pain pump, and deep brain stimulator insertion [11-12]. Such protocols focus on multiple aspects of the psychosocial perspective, including existing psychiatric disease, substance use disorder, lifestyle, treatment adherence etc. In such protocols, each of these aspects is assessed and weighed independently for a specific risk deemed important. For instance, tobacco use disorder is weighed differently in a heart transplant candidate than in a liver transplant candidate whereas alcohol use disorder is of highest importance in a liver transplant candidate.

Suicidality or personality features may be of special importance in assessing a candidacy for the insertion of a left ventricular assist device.

Since no available guidelines for this particular population existed, we reviewed similar, established protocols for psychosocial risk assessment and focused on addictive disorders, 
psychiatric disorders, psychopathology, and other psychosocial risk factors during the initial, thorough consultation. Obviously, addictive disorders evaluation was the most important aspect of our psychosocial risk formulation when assessing the PICC line compliance. A comprehensive addiction history was obtained focused not only on the primary drug of choice but also other substance use patterns. As expected from standard addiction assessment, the patient was questioned about drug of choice, active use, route of use, longest period of abstinence, previous chemical dependency treatments, relapse prevention skills, and involvement in Alcoholics Anonymous (AA) or Narcotics Anonymous (NA). Insight level was assessed of the person's addiction and motivation for treatment. After the diagnosis was given, appropriate addiction treatment recommendations were offered in accordance with American Society of Addiction Medicine (ASAM) guidelines [14-15]. Because of the medical acuity in most cases, recommended addiction treatment was either deferred until the end of the antimicrobial course or, in case the decision was made to keep the patient in the hospital, regular follow-up visits by the same psychiatric consultant were completed throughout the hospital stay. During those visits, a dual diagnosis approach was adopted: psychiatric comorbidities were noted and treated where necessary and addiction counseling given. Social support systems, general treatment adherence, living circumstances and financial situation were noted as part of the assessment.

\section{Risk categorization}

The retrospective case review was performed. A psychosocial risk profile was formulated for each case and was assigned a risk category of high (HR), moderate (MR), or low (LR) for a peripherally inserted central catheter (PICC) for OPAT.

\section{High risk}

Between $72 \%$ to $97 \%$ of all opioid-addicted persons prefer the oral route for drug administration [16]. Use of an intravenous route is associated with most severe forms of addictive disorders [17-18]. Therefore, all active intravenous drug users within 12 months of the need for OPAT were deemed at high risk for PICC violation without exception. Patients with remote use of intravenous drugs who were in sustained remission but currently deemed at high relapse risk were also assigned to this group.

Twelve months was the length of required abstinence chosen for determining high-risk candidates based on the following considerations. Most similar existing protocols rely on very limited data on relapse risk prediction, based on a preceding abstinence period [19-22]. We reviewed the literature on all substance use disorders to determine the ideal period of abstinence that would be a reliable indicator for ongoing sobriety. Six months up to two years has been widely considered as the period of required abstinence as relapse predictors in different protocols. Considering our population, we decided on 12 months based on the limited literature and anecdotal data [22].

Only 12 months of abstinence from IV substance use disorder without history of formal addiction treatment was adequate to avoid the high risk category but we also carefully looked at individuals who were at high risk of relapse due to a variety of psychosocial factors. To determine high relapse risk in individuals with sustained remission from their addictive disorder, the following factors were considered, most of which are also part of ASAM dimensional assessments [14]: not actively involved in a sober support group (Narcotics Anonymous or Alcoholics Anonymous), active environmental risk factors for relapse (eg, ongoing association with active intravenous drug users), history of recurrent chemical dependency treatment failure, lack of insight or fundamental understanding of addiction, active psychiatric co-morbidity, poor support system and poor financial situation. When unable to confirm the active IVDU or when challenged with poor cooperation, the ID specialist 
weighed the likelihood that the current clinical infectious situation was caused by active IVDU. When the ID specialist deemed the likelihood high, the case was assigned to the HR group regardless of confirmed use within 12 months. Other considerations factored into the risk profile, including active addiction to other substances (intravenous and non-intravenous patterns), active psychosocial issues (eg, poor support system, poor financial situation), history of poor compliance, and active psychiatric issues. In summary, any patient with a history of IVDU disorder in remission, who had acute concerns for other addictive disorders, psychiatric disorders, or psychosocial issues, was still regarded at high risk for PICC violation.

The HR group was either not approved for discharge from the hospital with a PICC line or were approved to be discharged to a nursing home, a long-term, acute care setting, or an ambulatory infusion center where daily peripheral line insertion is available for all days that therapy is needed. In the rural setting, the decision to keep the HR group in the hospital for the entire course of antimicrobial treatment was made frequently because alternative options were not available. Options such as ambulatory care centers and social services, including transportation, were non-existent in the residential towns of many patients. Patients who involuntarily were unable to adhere to the recommended plan were allowed to be discharged against medical advice. For some severe cases, civil commitment was considered but the local administration was not supportive of this route because the judicial mechanisms to support this option were limited in that particular state.

\section{Moderate risk}

Patients were assigned to the MR group if they had a history of intravenous drug use that was in remission for at least 12 months or they had no history of intravenous drug use but had an active opioid, cocaine, or methamphetamine use disorder. The rationale of this assignment was based on the recognition of an untreated addictive disorder, with the possibility of relapse and escalation to an intravenous form of abuse with the availability of the PICC line [23]. Three substance categories received primary focus - cocaine, methamphetamine, and opioids - for this group because these substances are most commonly abused in IVDU [24]. Alcohol or cannabis use disorders were included when they were in severe active form. This rationale was not based on risk of violation of the PICC line but on risk of non-compliance or increased risk of ID relapse during treatment because of active, severe addiction.

The MR group was approved for discharge from the hospital with a PICC line under certain conditions. Patients were allowed to leave with a PICC line but received antimicrobial injections in an ambulatory center at the hospital. For these patients, urine drug screens were randomly conducted. Strict adherence with ambulatory care was required as well as compliance with psychiatry and ID follow-up appointments.

\section{Low risk}

Patients without a history of intravenous drug use and in full remission from any other addictive disorder (excluding alcohol or cannabis use disorders in mild or moderate degree) for at least 12 months were assigned to the LR group. This group was cleared for transitioning to OPAT with a PICC line and to routine ID and psychiatric care.

\section{Results}

Between January 2014 and July 2016, 20 cases were identified that were seen by ID services and referred to psychiatry consultation-liaison services for risk assessment and disposition planning (Table 1). Ten cases were assigned to the HR category; five cases, the MR category; and five cases, the LR category. The average age for HR was 38.6 years; for MR, 40.8 years; and for LR, 49.2 years. Of the 20 cases, 12 (60\%) were men and eight (40\%) were women; in the HR 


\section{Cureus}

group, seven were men; the MR group, three were women; and in the LR group, three were men. The mean recommended days of antimicrobial therapy for the HR group was 40.2 days (median [range], 42 \{10-56\} days); for the MR group, 32.2 (median [range], 21 \{14-42\} days); and for the LR group, 28 (median [range], 28 \{14-70\}).

\begin{tabular}{|c|c|c|}
\hline $\begin{array}{l}\text { Risk } \\
\text { Category }\end{array}$ & Description & Recommendation \\
\hline High & $\begin{array}{l}\text { Active or suspected IV drug use within } 12 \\
\text { months without exception; or drug use in } \\
\text { sustained remission >=12 months but } \\
\text { currently at high relapse risk. }^{a}\end{array}$ & $\begin{array}{l}\text { No approval for discharge home with a PICC line; where } \\
\text { possible, discharged to a nursing home, rehabilitation setting, } \\
\text { or long-term acute care facility or kept in the hospital for the } \\
\text { entire antimicrobial course; if involuntary, discharged home } \\
\text { against medical advice. }\end{array}$ \\
\hline Moderate & $\begin{array}{l}\text { No history of IV drug use but active } \\
\text { addiction to methamphetamine, cocaine, } \\
\text { opioids, alcohol (only when severe), or } \\
\text { cannabis (only when severe). }\end{array}$ & $\begin{array}{l}\text { Approved for discharge from the hospital under restricted } \\
\text { conditions: administration of antimicrobial therapy in the } \\
\text { hospital ambulatory unit daily with random UDS and strict } \\
\text { adherence to recommended psychiatric and ID follow-up } \\
\text { appointments. }\end{array}$ \\
\hline Low & $\begin{array}{l}\text { No history of IV drug use and in full } \\
\text { remission from methamphetamine, cocaine, } \\
\text { opioids, or active alcohol or cannabis use } \\
\text { disorder, or a combination, only of mild or } \\
\text { moderate severity. }\end{array}$ & $\begin{array}{l}\text { Cleared for OPAI with a PICC line and appropriate follow-up } \\
\text { appointments. No routine UDS required. }\end{array}$ \\
\hline
\end{tabular}

\section{TABLE 1: Psychosocial Risk Formulation for OPAT among IV Drug Use Histories}

Abbreviations: ID, infectious disease; IV, intravenous; OPAT, outpatient antimicrobial therapy; PICC, peripherally inserted central catheter; UDS, urinary drug screen.

a. See text for indicators of high relapse risk.

In the HR group, an average of $80.3 \%$ of IVDU patients completed the antimicrobial therapy days as recommended by ID service; in the MR group, 90.6\%; and in the LR group, 100\% (Table 2). The most common ID diagnosis for the HR group was native valve endocarditis, followed by epidural abscess, diskitis, facial abscess, and pneumonia. For MR and LR groups, the ID diagnoses were more equally distributed among thigh abscess, septic arthritis, joint arthritis, meningitis, and osteomyelitis. 


\section{Cureus}

\begin{tabular}{|c|c|c|c|c|c|}
\hline $\begin{array}{c}\text { Risk } \\
\text { Category }\end{array}$ & $\begin{array}{l}\text { No. of } \\
\text { Patients } \\
(\mathrm{M} / \mathrm{F})\end{array}$ & $\begin{array}{l}\text { ID } \\
\text { Diagnosis, } \\
\text { No. }\end{array}$ & $\begin{array}{l}\quad \text { Age, } \\
\text { Mean, } \\
\text { Median, y }\end{array}$ & $\begin{array}{l}\text { Recommended for } \\
\text { Therapy, Mean, Median, d }\end{array}$ & $\begin{array}{l}\text { Compliance With Therapy, } \\
\text { Mean (\%), Median, d }\end{array}$ \\
\hline High & $10(7 / 3)$ & $\begin{array}{l}\text { Endocarditis, } \\
5 \\
\text { Epidural } \\
\text { abscess, } 2 \\
\text { Diskitis, } 1 \\
\text { Fascial } \\
\text { abscess, } 1 \\
\text { Pneumonia, } 1\end{array}$ & $38.6,42$ & $40.2,42$ & 32.3 (80.3), 42 \\
\hline Moderate & $5(2 / 3)$ & $\begin{array}{l}\text { Thigh } \\
\text { abscess, } 1 \\
\text { Septic } \\
\text { arthritis, } 1 \\
\text { Pneumonia, } 1 \\
\text { Prosthetic } \\
\text { joint arthritis, } \\
1 \\
\text { Osteomyelitis, } \\
1\end{array}$ & $40.8,34$ & $32.2,21$ & 29.4 (90.6), 21 \\
\hline Low & $5(3 / 2)$ & $\begin{array}{l}\text { Endocarditis, } \\
1 \\
\text { Meningitis, } 1 \\
\text { Soft tissue } \\
\text { infection, } 2 \\
\text { MSSA } \\
\text { septicemia, } 1\end{array}$ & $49.2,52$ & 28,28 & 28 (100), 28 \\
\hline
\end{tabular}

TABLE 2: Risk Category Demographic Characteristics and ID Diagnosis and Antibiotic Therapy Compliance

Abbreviations: F, female; ID, infectious disease; M, male; MSSA, methicillin-sensitive Staphylococcus aureus.

In the HR group, the most common addictive disorder was intravenous opioid use disorder, which comprised $80 \%$ of all HR cases, followed by intravenous cocaine and methamphetamine use disorders (Table 3). Of the 10 patients in the HR group, four (40\%) had relapses on the drug of choice during treatment. Among these four patients, two relapsed in the hospital, one in a 


\section{Cureus}

nursing home, and one following discharge against medical advice. Only one patient in the MR group had a relapse following discontinuation of treatment in an outpatient setting. Violation of the PICC line per se could not be determined. No LR patient had a relapse while receiving OPAT.

\begin{tabular}{|c|c|c|c|c|}
\hline Risk Category & Patients, No. & Primary Drug of Choice, No. & Relapse During Treatment & Relapse Venue \\
\hline \multirow{3}{*}{ High } & \multirow{3}{*}{10} & Opioids, 8 & \multirow{3}{*}{4} & Hospital, 2 \\
\hline & & Methamphetamine, 1 & & Nursing home, 1 \\
\hline & & Cocaine, 1 & & AMA discharge, 1 \\
\hline \multirow{3}{*}{ Moderate } & \multirow{3}{*}{5} & Opioids, 2 & \multirow{3}{*}{1} & \\
\hline & & Cocaine. 2 & & Home \\
\hline & & Cannabis, 1 & & \\
\hline \multirow{3}{*}{ Low } & \multirow{3}{*}{5} & Opioids, 3 & \multirow{3}{*}{0} & \\
\hline & & Cocaine, 1 & & \\
\hline & & Alcohol, 1 & & \\
\hline
\end{tabular}

TABLE 3: Risk Category and Intravenous Drug Use During Antibiotic Treatment Abbreviation: AMA, against medical advice

Four of the 20 patients received more than one diagnosis of an addictive disorder according to DSM-V. These data are detailed in Table 4.

\begin{tabular}{|c|c|c|c|c|c|c|}
\hline \multirow[b]{2}{*}{ Risk Category } & \multicolumn{6}{|c|}{ Use Disorder, No. of Patients } \\
\hline & Opioid & Amphetamine & Cocaine & Cannabis & Alcohol & Sedative \\
\hline High & 8 & 1 & 3 & 2 & 2 & 3 \\
\hline Moderate & 4 & 0 & 2 & 2 & 0 & 3 \\
\hline Low & 2 & 1 & 1 & 0 & 3 & 0 \\
\hline Total & 14 & 2 & 6 & 4 & 5 & 6 \\
\hline
\end{tabular}

TABLE 4: Risk Category and Diagnostic and Statistical Manual of Mental Disorders (Fifth Edition) Addictive Disorder Diagnoses

\section{Discussion}


An underlying disorder such as IVDU may lead to ID or an ID may occur unrelated to the addiction. Management of these infections in the IVDU population is a challenge, especially in rural settings with limited resources [5]. One prospective observational study conducted in an urban university hospital in Singapore assessed the care of patients with IVDU who required outpatient intravenous antibiotic therapy [25]. Tamper-proof PICC catheters were used successfully in IVDU patients who self-reported drug use. However, the severity of addiction was not delineated.

Our study is a retrospective review of an ID practice from January 2014 through July 2016 that used an addiction specialist and psychiatrist to aid in the clinical decision- making for the discharge of infected IVDU patients who needed OPAT. Discharge options in the rural setting were scarce. Rarely are board-certified specialists in these two disciplines simultaneously practicing in a small, rural town. The objective of this review was to determine whether a risk stratification criteria aid could be developed to determine who could more safely be discharged home with OPAT. If criteria could be created to differentiate patients who could safely be discharged with OPAT by specialists in a rural community, these criteria could be further tested in a randomized, prospective manner among IVDU persons who were infected and needed OPAT, to aid non-specialist providers in rendering care to this population in small US towns.

We previously reported the cost of hospital care for a recalcitrant IVDU patient who was successfully treated for the infection each time, but because the original problem of intravenous drug addiction was not manageable, multiple new infections resulted [26]. The high non-compliance rate in abstinence from drug use despite being hospitalized in a rural community (the only option available), in the context of the high cost for such care for this population, highlights the gravity of the rural situation and the monumental effect it has on rural hospital finances [27-28]. Our goal was to aid health care providers with a tool to most effectively use limited resources safely. Other disciplines such as organ transplant services allocate resources in an analogous fashion.

We found that the HR group had a greater likelihood of noncompliance with OPAT completion as well as subsequent illicit drug use during that timeframe than the MR and LR groups. Use of illicit drugs during hospitalization occurred despite treatment recommendations for the patient's addictive disorder and follow-up visits by the psychiatrist during the hospital stay.

All these patients had a life-threatening ID that required parenteral antibiotic treatment. No primary residential addiction program in the area considered them candidates for the addiction programs because of their medical acuity. Use of illicit drugs while being seen by a psychiatrist stresses the degree of the patient's addiction and the importance of securing the correct psychiatric or addiction diagnosis, or both. This finding highlights that until the primary disorder, addiction, is managed successfully, treatment of secondary problems such as infections is handicapped. Hundred per cent compliance is critical in completing OPAT and not using illicit intravenous drugs during the OPAT timeframe as seen in the LR group.

Unfortunately, the numbers in this review are small, a limitation of this study, and yet another reality of reporting from small US towns.

The HR group had more life-threatening infections than the LR group. This fact accounts for the shorter durations of OPAT recommended by ID services. However, it is unlikely to account for the non-existent illicit drug use in the LR group. Opioid use existed in all three risk categories, which emphasizes that the active degree of addiction is the probable predictor of compliance in therapy and safety in OPAT administered via a PICC. The predictor of safety in OPAT discharge likely lies in the adequacy of obtaining an accurate drug use history by a person trained in securing such histories. Many investigators have shown [29-30] that internists and family practitioners often miss the addiction history, let alone accurately determining the degree or 
severity of the addiction. In this study, two specialists (ID specialist and addiction psychiatrist) simultaneously practiced in a rural community, which is a rare situation. Identification of the risk behavior and its degree are pivotal delineators in developing risk stratification criteria.

\section{Conclusions}

This study provides a risk stratification aid to select those who could safely be discharged with OPAT in US small towns. The severity of the addictions in the HR group and their use of drugs during the treatment period emphasize the enormity of their plight and the lack of options for their care. The proposed HR, MR, and LR risk stratifications of IVDU patients who require OPAT needs further prospective testing with significant numbers to validate the criteria. Such risk stratification of IVDU patients in need of OPAT would afford safer allocation of limited resources, especially in rural locations.

\section{Additional Information}

\section{Disclosures}

Human subjects: Consent was obtained by all participants in this study. Mayo Clinic IRB issued approval 17-002887. Principal Investigator Notification: The above-referenced application is approved by expedited review procedures ( 45 CFR 46.110, item 5). This approval is valid for a period of three years. The reviewer conducted a risk-benefit analysis and determined that the study constitutes minimal risk research. The reviewer determined that this research satisfies the requirements of $45 \mathrm{CFR} 46.111$. The reviewer approved the waiver of the requirement to obtain informed consent in accordance with 45 CFR 46.116, as justified by the investigator, and waiver of Health Insurance Portability and Accountability Act of 1996 (HIPAA) authorization in accordance with applicable HIPAA regulations. The reviewer approved the accrual of 30 subjects and to review data that exists between January 1, 2005, and December 31, 2016. Mayo Clinic Institutional Reviewer. Animal subjects: All authors have confirmed that this study did not involve animal subjects or tissue. Conflicts of interest: In compliance with the ICMJE uniform disclosure form, all authors declare the following:

Payment/services info: All authors have declared that no financial support was received from any organization for the submitted work. Financial relationships: All authors have declared that they have no financial relationships at present or within the previous three years with any organizations that might have an interest in the submitted work. Other relationships: All authors have declared that there are no other relationships or activities that could appear to have influenced the submitted work.

\section{References}

1. Goenaga Sanchez MA, Millet Sampedro M, Garde Orbaiz C, Carrera Macazaga JA: About the guidelines for outpatient parenteral antimicrobial therapy. Clin Infect Dis. 2004, 1:1730-1731. 10.1086/425507

2. Hopper JA, Shafi: Management of the hospitalized injection drug user . Infect Dis Clin North Am. 2004, 39:571-587. 10.1016/S0891-5520(02)00009-0

3. Papalekas E, Patel N, Neph A, Moreno D, Zervos M, Reyes KC: Outpatient parenteral antimicrobial therapy (OPAT) in intravenous drug users (IVDUs): epidemiology and outcomes. Open Forum Infect Dis Dec. 2014, 1:S52-S53.

4. Hempel, S, Gibbons, MM, Ulloa, JG et al. : Rural healthcare workforce: a systematic review. Department of Veterans Affairs (US), Washington, DC; 2015.

5. Pullen E, Oser C: Barriers to substance abuse treatment in rural and urban communities: counselor perspectives. Subst Use Misuse. 2014, 49:891-901. 10.3109/10826084.2014.891615

6. Robinson LR, Holbrook JR, Bitsko RH, Hartwig SA, Kaminski JW, Ghandour RM: Differences in health care, family, and community factors associated with mental, behavioral, and developmental disorders among children aged 2-8 years in rural and urban areas: United States. MMWR. 2017, 66:1-11. 
7. Stockwell S: Rural pregnant women and newborns hit hard by opioid crisis . Am J Nurs. 2017, 117:10.1097/01.NAJ.0000513278.76259.6d

8. Shannon LM, Havens JR, Hays L: Examining differences in substance use among rural and urban pregnant women. Am J Addict. 2010, Nov-Dec. 10.1111/j.1521-0391.2010.00079.x

9. Monnat SM, Rigg KK: Examining rural/urban differences in prescription opioid misuse among US adolescents. J Rural Health. 2016, 32:204-218. 10.1111/jrh.12141

10. Havens JR, Oser CB, Leukefeld CG, et al.: Differences in prevalence of prescription opiate misuse among rural and urban probationers. Am J Drug Alcohol Abuse. 2007, 33:309-17. 10.1080/00952990601175078

11. Maldonado JR, Dubois HC, David EE, et al.: The Stanford integrated psychosocial assessment for transplantation (SIPAT): a new tool for the psychosocial evaluation of pre-transplant candidates. Psychosomatics. 2012, 53:123-132. 10.1016/j.psym.2011.12.012

12. Yost GL, Bhat G, Ibrahim KN, Karountzos AG, Chandrasekaran M, Mahoney E: Psychosocial evaluation in patients undergoing left ventricular assist device implantation using the transplant evaluation rating scale. Psychosomatics. 2016, 57:41-46.

10.1016/j.psym.2015.07.013

13. American Psychiatric Association: Diagnostic and statistical manual of mental disorders: DSM-5. 5th ed. American Psychiatric Publishing, Arlington, VA; 2013.

14. McKay JR, Cacciola JS, McLellan AT, Alterman AI, Wirtz PW: An initial evaluation of the psychosocial dimensions of the American Society of Addiction Medicine criteria for inpatient versus intensive outpatient substance abuse rehabilitation. J Stud Alcohol. 1997, 58:239-252. 10.15288/jsa.1997.58.239

15. American Society of Addiction Medicine (ASAM) . (2017). Accessed: May 19: http://www.asam.org/.

16. Kirsh K, Peppin J, Coleman J: Characterization of prescription opioid abuse in the United States: focus on route of administration. J Pain Palliat Care Pharmacother. 2012, 26:348 -361.

17. Oldendorf WH: Some relationships between addiction and drug delivery to the brain. NIDA Res Monogr. 1992, 120:13-25.

18. Mars SG, Bourgois P, Karandinos G, Montero F, Ciccarone D: “Every 'never’ I ever said came true”: transitions from opioid pills to heroin injecting. Int J Drug Policy. 2014, 25:257-266. 10.1016/j.drugpo.2013.10.004

19. Karim Z, Intaraprasong P, Scudamore CH, et al.: Predictors of relapse to significant alcohol drinking after liver transplantation. Can J Gastroenterol. 2010, 24:245-250.

20. Chalana H, Kundal T, Gupta V, Malhari AS: Predictors of relapse after inpatient opioid detoxification during 1-year follow-up. J Addict. 2016, 10.1155/2016/7620860

21. Schneekloth TD, Biernacka JM, Hall-Flavin DK, et al.: Alcohol craving as a predictor of relapse. Am J Addict. 2012, 21: S20-S26. 10.1111/j.1521-0391.2012.00297.x

22. Schneekloth TD: Mayo clinic transplant center psychosocial guidelines. Schneekloth TD (ed): Mayo Clinic, Rochester, Minnesota; 2017.

23. Ferraris VA, Sekela ME: Missing the forest for the trees: the world around us and surgical treatment of endocarditis. J Thorac Cardiovasc Surg. 2016, 152:677-680.

10.1016/j.jtcvs.2016.05.014

24. Commonly Abused Drugs Charts . (2017). Accessed: May 19: https://www.drugabuse.gov/drugs-abuse/commonly-abused-drugs-charts.

25. Ho J, Archuleta S, Sulaiman Z, Fisher D: Safe and successful treatment of intravenous drug users with a peripherally inserted central catheter in an outpatient parenteral antibiotic treatment service. J Antimicrob Chemother. 2010 , 65:2641-2644. 10.1093/jac/dkq355

26. Libertin CR, Camsari UM, Hellinger WC, Schneekloth TD, Rummans TA: The cost of a recalcitrant intravenous drug user with serial cases of endocarditis: Need for guidelines to improve the continuum of care. IDCases. 2017, 8:3-5. 10.1016/j.idcr.2017.02.001

27. Fleischauer AT, Ruhl L, Rhea S, Barnes E: Hospitalizations for endocarditis and associated health care costs among persons with diagnosed drug dependence: North Carolina, 2010-2015. MMWR. 2017, 66:569-573. 10.15585/mmwr.mm6622a1

28. Hartman L, Barnes E, Bachmann L, Schafer K, Lovato J, Files DC: Opiate injection-associated infective endocarditis in the southeastern United States. Am J Med Sci. 2016, 352:603-608. 10.1016/j.amjms.2016.08.010

29. Tenegra JC, Leebold B: Substance abuse screening and treatment. Prim Care. 2016, 43:217227. 10.1016/j.pop.2016.01.008 


\section{Cureus}

30. The National Center on Addiction and Substance Abuse at Columbia University: Missed Opportunity: National Survey of Primary Care Physicians and Patients on Substance Abuse. Columbia University \& Josiah Macy, Jr. Foundation, New York, NY; 2000. 